\title{
EL RIESGO DE MERCADO Y LA ESTRUCTURA INTERTEMPORAL DE LAS TASAS DE INTERÉS
}

\author{
Joaquín Tapia Maruri* \\ Secretaría de Agricultura, Ganadería, Desarrollo Rural, Pesca y Alimentación
}

(Recibido 23 de septiembre de 2002, aceptado 11 de febrero de 2003)

\section{Resumen}

Este trabajo desarrolla un modelo para la determinación conjunta del riesgo de mercado (tipo de cambio, inflación y tasas de interés) y su interrelación con la estructura intertemporal de las tasas de interés. Con el objeto de identificar variables de política económica que afectan el riesgo de mercado y la estructura de las tasas de interés, en un contexto de expectativas racionales, el trabajo realiza un análisis a partir de la teoría cuantitativa del dinero.

\begin{abstract}
This paper develops a model for the joint determination of the risk of market (exchange rate, inflation rate and interest rate) and its relationship with the intertemporal structure of the interest rates. In order to identify variables of economic policy that affect market rate and the structure of interest rates, in a context of rational expectations, the paper carries out an analysis using quatitative theory of money.
\end{abstract}

Clasificación JEL: E43, E60

Palabras clave: Expectativas racionales, Tasa de interés, Riesgo de mercado

* Avenida Insurgentes Sur 489, piso 12. Col. Hipódromo Condesa. Teléfono: 5271-5873. Correo electrónico: jtapia.sfa@sagarpa.gob.mx

Las opiniones vertidas en este documento, así como los errores y omisiones, son responsabilidad exclusiva del autor. El autor agradece los valiosos comentarios de dos árbitros anónimos, Arnold Zellner, Bernardo González-Aréchiga, Víctor Guerrero, Javier Salas y Juan Carlos Cortés, así como el apoyo brindado por Alejandro Cavazos, Jesús Rodríguez y la asistencia técnica y de investigación proporcionada por María Fernanda González Sardina y Francisco Rivero. 


\section{Introducción}

La elaboración de un modelo econométrico para medir el riesgo de mercado, tomando como referente el comportamiento de las tasas de interés, es útil para cálcular el valor estimado o mean behavior de dicha variable de referencia, así como para realizar pruebas de naturaleza VaR. Al analizar los residuales generados por dicho modelo, debemos ser capaces de medir el riesgo de mercado y establecer intervalos de confianza para determinar el "Value at Risk" de las posiciones cortas y largas de los intermediarios financieros. Lo anterior, bajo el supuesto de la existencia de normalidad.

En general, este tipo de modelos son construidos y usados por las autoridades monetarias, para decidir si se debe modificar o no la estructura intertemporal de las diferentes tasas de interés. Esto último a través de cambios inducidos en la tasa de inflación, en el tipo de cambio esperado o en la oferta monetaria, entre otras variables de control. No obstante, el desarrollo de este tipo de modelos, con frecuencia, resulta ser bastante complicado.

Recordemos que en la historia reciente de México se han presentado diversas crisis financieras caracterizadas, entre otras cosas, por un comportamiento inestable de las tasas de interés. Por ejemplo, durante el mes de abril del año de 1995 , se registraron niveles de tasas de interés de $75.0 \%$ promedio mensual, con un tipo de cambio de $\$ 6.70$ pesos por dólar (al cierre de ese mes) y una inflación anualizada de $29.4 \%$. Años más tarde, la crisis económica-financiera de los países asiáticos, iniciada en julio de 1997, influyó en la trayectoria de las tasas de interćs domésticas llevándolas a un nivel en $20.2 \%$, mientras que el tipo de cambio promedio, en ese mes, fue de $\$ 8.30$ pesos por dólar y la inflación de 19.7\%. Para el último trimestre de 1998, las crisis de Rusia y Brasil repercutieron una vez más en el comportamiento de las variables económicas de nuestro país. En septiembre de ese año, las tasas de interés se situaron en $40.8 \%$ y el tipo de cambio alcanzó $\$ 10.15$ pesos por dólar, con una inflación de $16.0 \%$, la cual fue de $18.6 \%$ para el mes de diciembre.

Por todo lo anterior, el objetivo principal del presente trabajo es la construcción de un modelo, capaz de describir el comportamiento de las tasas de intcrés y su estructura intertemporal. Su desarrollo se expone en la primera parte de este trabajo. En la segunda y tercera, se especifican los modelos a estimar y se analizan los resultados alcanzados. Finalmente, se presentan las conclusiones y se incluyen cuatro apéndices.

\section{El modelo}

Sabemos que la teoría cuantitativa del dinero asocia a la demanda y a la oferta agregada a través de la siguiente condición de equilibrio:

$$
M V=P Y,
$$

donde $M$ es la cantidad de dinero en circulación; $V$ es la velocidad de circulación del dinero; $Y$ es el valor del producto interno bruto y $P$ es el nivel general de precios. ${ }^{1}$ Además, este enfoque supone la existencia de stocks y flujos de equi-

1 A esta teoría se le conoce como la Teoría General de Precios, la cual ha servido como base para la construcción de los modelos de inflación basados en el supuesto de que el dinero es una fuente potencial del fenómeno inflacionario. 
librio, así como el intercambio de bienes, servicios y activos entre los diferentes agentes económicos.

La ecuación número uno establece que con el dinero existente en circulación dentro de la economía se compra la producción actual del mercado y, a través de este mecanismo, los stocks se asocian a la demanda agregada y los flujos a la oferta agregada. Sin embargo, esta igualdad o "ecuación de intercambio" se transforma en la "ecuación de Cambridge" haciendo alusión a la demanda de dinero, al poder de compra de éste en términos reales con relación al ingreso y a la estabilidad de la velocidad del dinero, de acuerdo a las siguiente relación de comportamiento:

$$
M / P=m^{d}=k Y \text {, }
$$

donde $k=1 / V$ es el inverso de la velocidad de circulación, la cual es una relación estable que depende de los hábitos de pago de los agentes y de la tasa de interés. En este sentido, la demanda de saldos monetarios reales $\left(m^{d}\right)$ es una fracción del ingreso $(Y)$.

\subsection{Demanda de dinero reconsiderada}

$\mathrm{Si}$ aceptamos que el dinero representa un activo dentro de una cartera, debemos considerar aquellas variables que lo afectan tanto en sus costos como en sus rendimientos derivados de su propiedad, así como el factor de incertidumbre si hablamos de u1n análisis de portafolio. En este sentido, la demanda de dinero deberá incluir aquellos términos que representen el rendimiento esperado $\left(r_{d}\right)$ y corriente de otros activos, con el fin de permitir su sustitución $\left(r_{s}\right)$ y/o su complementariedad $\left(r_{c}\right)$. También se puede especificar la sustitución con bienes aproximada con la inflación $(\pi)$, incluir el efecto riqueza $(w)$ y el efecto ingreso $(y)$. De esta forma, la demanda de dinero se puede representar de la siguiente manera:

$$
m^{d}=f\left(y, w, r_{d}, r_{s}, r_{c}, \pi\right)
$$

\subsection{Demanda de dinero en una economia semi-abierta}

Cabe la posibilidad de incorporar el hecho de que en la actualidad el proceso de integración de mercados financieros, a partir de la globalización y de la apertura financiera, permite incluir variables como el tipo de cambio nominal $(\phi)$, la tasa de interés externa $\left(r_{d}^{*}\right)$, y otros tipos de activos, de acuerdo a la sustitución $\left(r_{s}^{*}\right)$ y a la complementariedad $\left(r_{c}^{*}\right)$ deseada. $^{2}$ Asimismo, los bienes pueden ser un sustituto o complemento del dinero en términos de la inflación externa $(\mu)$, por lo que la demanda de dinero es igual a:

$$
m^{d}=f\left(y, w, r_{d}, r_{s}, r_{c}, \pi, \mu, r_{d}^{*}, r_{s}^{*}, r_{c}^{*}, \phi\right) .
$$

2 Aunque la imperfecta movilidad de capital y la existencia de mercados a futuro poco desarrollados invalidan la condición de la paridad de las tasas de interés, ayudan a la determinación de los montos y rendimientos de los activos. Véase, Tapia (1992). 
Es más, se pueden agregar spreads de tasas de interés internas $(\psi)$ o externas $(\zeta)$, o una combinación de ambas $(\lambda)$, como una medida imperfecta de incertidumbre. De tal manera que la demanda de dinero quede determinada de la siguiente manera:

$$
m^{d}=f\left(y, w, r_{d}, r_{s}, r_{c}, \pi, \mu, r_{d}^{*}, r_{s}^{*}, r_{c}^{*}, \phi, \psi, \zeta, \lambda\right) .
$$

Esta especificación de la demanda de dinero en una economía semi-abierta añade dos elementos: ${ }^{3}$ una mejor especificación y una mayor estabilidad.

\subsection{Determinación de la tasa de interés}

En estas condiciones, la demanda de dinero puede determinar las tasas de interés en términos de variables como el ingreso, la riqueza y los rendimientos esperados y corrientes de otros activos, tanto internos como externos; determinando de la misma forma factores de sustitución y complementariedad, no sólo con activos, sino también con bienes internos y externos. Por tal motivo, se incluye a los mercados de bienes, dinero y bonos, internos y externos, con el de fin de enmarcar su determinación en un contexto de equilibrio general. Para mayor detalle véase el Apéndice A, donde aparece la forma estructural del modelo.

Por otra parte, la especificación de este tipo de función de demanda de dinero, podría interpretarse como una demanda de final de período, en donde la condición de equilibrio se alcanza al término del período $t$, o al principio del $t+1$. Esto debido a que los choques no anticipados sobre las variables incluidas durante el intervalo de tiempo entre $t$ y $t+1$, permiten ajustar la trayectoria hacia el nuevo equilibrio, recolectando la información recibida y procesándola posteriormente; lo cual se hace explícito con la inclusión de un variable que mide la velocidad de ajuste. ${ }^{4}$

\subsection{Estructura intertemporal de tasas de interés, EITI}

Los inversionistas mantienen los bonos por el rendimiento total que pueden obtener. En este caso un bono cupón cero (BCC) genera rendimientos a través de dos fuentes: el pago de intereses y la ganancia de capital esperada. El rendimiento del bono, $r$, se obtiene como porcentaje del valor del cupón, $c$, entre el precio del bono, $P$, es decir:

$$
r=c / P \text {. }
$$

y

$$
P=c / r \text {. }
$$

Por otro lado, debido a que la ganancia esperada de capital, $g$, es la tasa de crecimiento esperada del precio del bono, tenemos que:

$$
g=\left(P^{e}-P\right) / P .
$$

3 Para una discución más profunda de esta especificación, véase a Edwards (1989).

4 Véase Tapia (1990). 
Esta ganancia se puede transformar en términos de tasas de interés esperadas y corrientes, dado que,

$$
r^{e}=c / P^{e},
$$

entonces

$$
g=\left(\left(c / r^{e}\right)-(c / r)\right) /(c / r) .
$$

Si se simplifica, tenemos que

$$
g=\left(r / r^{e}\right)-1 .
$$

De lo anterior, el rendimiento total, $e$, será la suma de la tasa de interés del mercado al momento de comprar el bono y la ganancia, o pérdida, de capital:

$$
e=r+\left(r / r^{e}\right)-1
$$

En términos del mercado, se define la tasa de interés como el rendimiento $r$, al vencimiento del tiempo $t$, durante el período $n$, que será liquidado en $t+n+1$ de la siguiente manera: $r_{t, n}$. El inversionista, entonces, recibirá el interés $r$, ganado en el período de tiempo $n$, del cupón $c$, más el valor del principal $B$. De esta manera la riqueza máxima acumulada, $V_{n}$, se define como

$$
V_{n}=c_{1}+B .
$$

Por otra parte, si el bono vence en el tiempo $n$ y los cupones tienen uno o varios cortes en el año, la cantidad máxima de riqueza, $V_{n}$, que puede ser acumulada al vencimiento del bono, puede ser a la par, sobre y bajo par con respecto a $\mathrm{B}$.

$$
V_{n}=c_{1}+c_{2}+c_{3}+\cdots+c_{n}+B .
$$

Si suponemos reinversión, es decir, $c_{1}<c_{2}<c_{3}<\cdots<c_{n}$, al término del período se tendrá que el valor actuarial es igual a:

$$
V_{n}=A\left(1+r_{t, n}\right)^{n}
$$

donde $A$ es el principal y

$$
A\left(1+r_{t, n}\right)^{n}=c_{1}+c_{2}+c_{3}+\cdots+c_{n}+B .
$$

Si se despeja $r_{t, n}$ se obtiene la siguiente ecuación,

$$
r_{t, n}=\sqrt[n]{(1 / A)\left(c_{1}+c_{2}+c_{3}+\cdots+c_{n}+B\right)}-1,
$$

o en su caso

$$
A=\frac{\left(c_{1}+c_{2}+c_{3}+\cdots+c_{n}+B\right)}{\left(1+r_{t, n}\right)^{n}} .
$$


La curva de rendimiento de los Certificados de la Tesorería de la Federación (Cetes) es fundamental, ya que representa un tipo de benchmark para la valuación de otros activos financieros realizada por los participantes del mercado. Generalmente, la EITI se construye en términos de plazos y rendimientos observados, es decir, un BCC a los diferentes plazos. También pueden agregarse obligaciones no convertibles porque estamos considerando un BCC. Lo anterior, en el sentido de que cada cupón tiene una maduración igual a su vencimiento, y en el caso del principal, igual al plazo de maduración; esto debido a que el valor de este activo es igual al valor de todos los componentes del BCC. En caso de que esta igualdad no se cumpla, se crearán oportunidades de arbitraje.

Para determinar el valor de cada $\mathrm{BCC}$ es necesario conocer el rendimiento y el plazo de vencimiento. A este rendimiento se le conoce como la tasa spot y a su comportamiento en los diferentes vencimientos se le conoce como la curva de rendimiento. La curva de rendimiento teórica juega un papel muy importante en la determinación del precio de todos los instrumentos financieros, ya que todos los activos de renta fija pueden verse como "cupón cero" y las obligaciones serán descontadas con la tasa libre de riesgo al plazo de referencia. La diferencia fundamental será entonces la exposición al riesgo crédito. Desde luego que las oportunidades de arbitraje se presentarán cuando los rendimientos actuales difieran de los teóricos y se realizaran a través del proceso conocido como stripping (separación de cortes de cupón para negociarse en el mercado secundario).

Es posible construir la curva de rendimiento teórica a partir de los rendimientos observados sobre los Cetes, de su cupón al vencimiento y del rendimiento observado de un conjunto de bonos cupón cero, en caso de no contar con algún rendimiento a plazo mayor. ${ }^{5}$ El rendimiento observado de un BCC se obtiene por medio implícito o equivalente, considerando el cupón y el plazo al vencimiento del BCC. En términos generales tenemos lo siguiente:

$$
V_{n}=c^{*} \sum_{t=1}^{n-1} \frac{1}{\left(1+r_{b t}\right)^{t}}+\frac{c^{*}+100}{\left(1+r_{b n}\right)^{n}},
$$

donde $c^{*}$ representa un corte de cupón del instrumento al plazo de vencimiento. Si se resuelve para $r_{b n}$,

$$
r_{b n}=\left[\frac{c^{*}+100}{V_{n}-c^{*} \sum_{t=1}^{n-1} \frac{1}{\left(1+r_{b t}\right)^{t}}}\right]^{1 / n}-1 .
$$

A lo anterior se le conoce con el nombre de "estructura intertemporal de tasas de interés" (EITI). Cabría añadir que los "instrumentos sintéticos" creados por los intermediarios, como por ejemplo los strips, no pueden ser añadidos a esta estructura intertemporal ya que: 1) son instrumentos con poca liquidez y reflejan un premio; 2) pueden existir sectores que deseen adquirir los instrumentos

5 Desde luego que se hace referencia a los rendimientos observados de bonos a la par, por lo que se le denomina curva de rendimiento a la par. 
sintéticos por el efecto del premio, distorsionando así la estructura intertemporal de la tasa de interés; y 3 ) el tratamiento fiscal que reciben los instrumentos sintéticos, el cual incide en el flujo anticipado de efectivo, se refleja en los rendimientos.

Ahora, es necesario establecer la relación entre las tasas spot, $r_{t}, y$ las forward, $r_{f}$, implícitas de plazo. ${ }^{6}$ En general, esta relación para $n$ periodos es igual a:

$$
r_{t, n}=\left\{\left(1+r_{1}\right)\left(1+r_{1,1}^{f}\right)\left(1+r_{1,2}^{f}\right)\left(1+r_{1,3}^{f}\right) \cdots\left(1+r_{t, n}^{f}\right)\right\}^{1 / t}-1,
$$

o inversamente

$$
f_{t, n}=\left\{\left(1+r_{t, n}\right)^{t+n} /\left(1+r_{n}\right)^{n}\right\}^{1 / t}-1 .
$$

Junto con esto, se estudia la interdependencia de un conjunto de tasas de interés en donde la sustitución de activos denominados en la misma moneda, con el mismo riesgo crédito, el mismo riesgo mercado y diferenciados únicamente por el plazo, se consideran instrumentos homogéneos. Este es el caso de los bonos cupón cero, donde la diferencia fundamental son los plazos involucrados, ya que estos valores gubernamentales no presentan ningún riesgo crédito y enfrentan el mismo riesgo mercado, como son las variaciones en la inflación o en el tipo de cambio.

Entonces, las estructuras de plazos producen un diferente impacto sobre los rendimientos de los activos o sobre lo que se conoce como la "convexidad" de la curva de rendimiento. Esto es el cambio en el precio de un bono ante cambios en la tasa de interés y se interpreta muchas veces como una varianza, pero es en realidad una elasticidad. Asimismo, afecta también a la duración por la tenencia de estos activos. ${ }^{7}$

La duración de un BCC es por definición igual a su plazo de vencimiento, ya que el pago de cupones o flujos de efectivo se lleva a cabo al vencimiento. En tales circunstancias, entre mayor sea el plazo al vencimiento, mayor será la duración y desde luego que a plazos mayores, mayor será la exposición a los riesgos de mercado $\mathrm{y} / \mathrm{o}$ volatilidad.

Tradicionalmente, la EITI asocia el rendimiento con los plazos al vencimiento de los diferentes activos, y ésta puede tener una pendiente horizontal, positiva o negativa, dependiendo de la percepción de los inversionistas sobre el futuro que seguirán las tasas de interés a diferentes plazos. ${ }^{8}$

6 Para mayor detalle, véase, Fabozzi (1993).

7 Véase, Livingston (1990).

8 Aunque se podría añadir, que las autoridades pueden inducir este cambio en la pendiente de la EITI como una medida para reducir los costos de ajuste del mercado. 
Figura 1. Estructura Anual de Tasas de Interés.

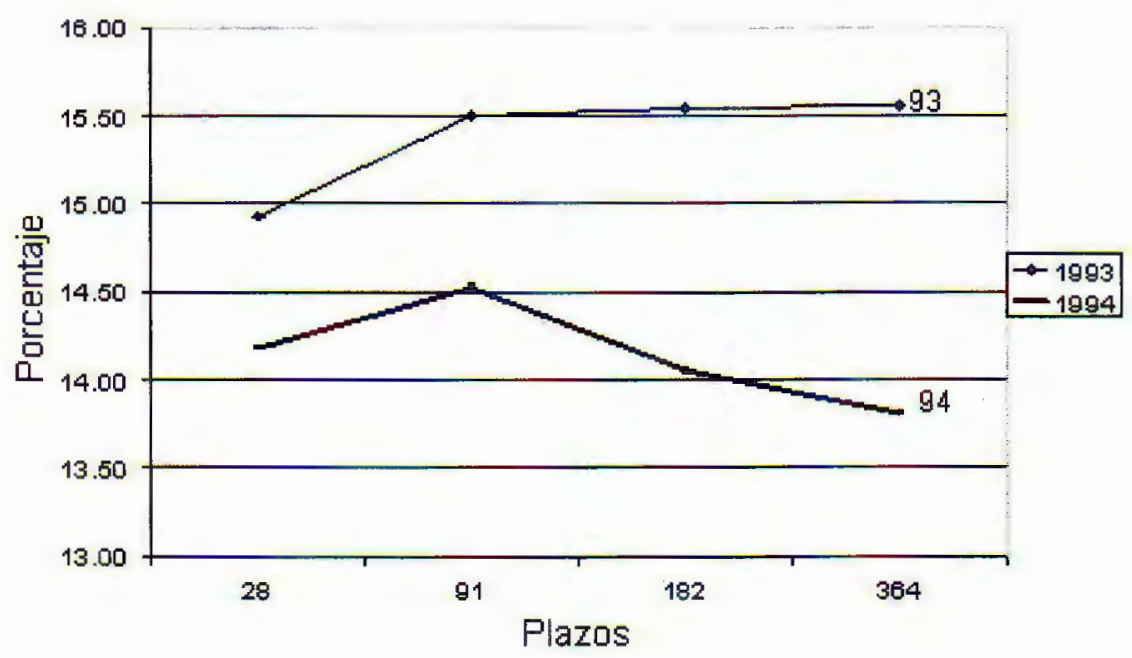

Figura 2. Estructura Anual de Tasas de Interés.




Figura 3. Estructura Anual de Tasas de Interés.

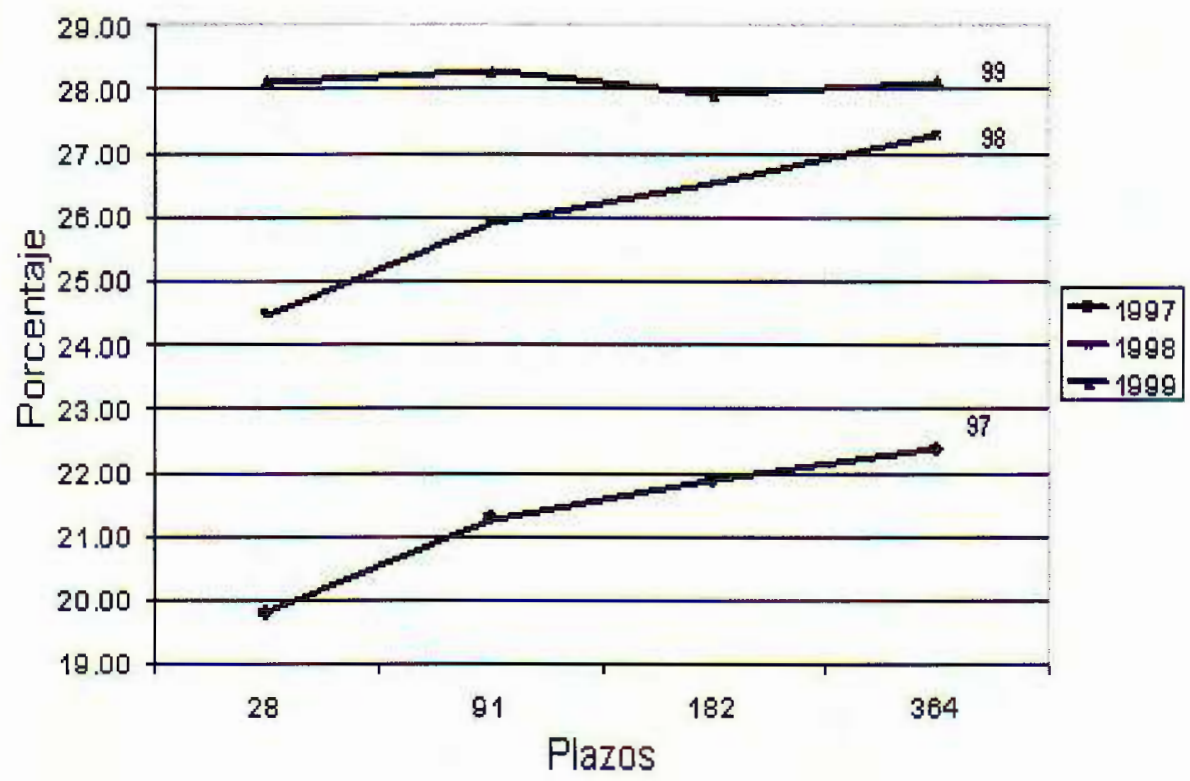

Las gráficas muestran la estructura intertemporal de las tasas de interés como un promedio anual. Se puede apreciar que en los años de crisis financiera como, 1994 y 1995, ésta se encuentra invertida, evidenciando con ello que a plazos mayores se esperaba una mayor estabilidad. Cabe mencionar, que en los años de 1997 y 1998 se mantuvo la estructura tradicional con pendiente positiva, en donde a mayor plazo, mayor rendimiento, dado el costo de oportunidad. Finalmente, en los años de 1993, 1996 y 1999 la EITI fue prácticamente horizontal, indicando expectativas invariantes o con alguna rigidez en el tiempo.

El análisis de la EITI se estudia más concretamente en la Teoría de las Expectativas de la Tasa de Interés (TETI) o en la de Segmentación de Mercados (TSM), también llamada Teoría de la Cobertura de la Estructura a Plazos (TCEP) y diversas variantes, las cuales se enunciarán en el apéndice D.

\section{Especificación del modelo}

Antes de especificar el modelo de la EITI, se estimará un modelo de "riesgo mercado" (tipo de cambio, inflación y tasa de interés) con el fin de ver su incidencia sobre las tasas de interés. Lo anterior, por considerar a estas variables fuentes fundamentales en la determinación estructural de la EITI. Desde luego, que para la estimación de los riesgos de mercado es necesario identificar al sistema de ecuaciones que conforman el modelo, por lo que en este caso se utilizaron las condiciones de orden. La especificación de cada una de las ecuaciones es la siguiente:

$$
K^{* *}>M^{* *}-1 \text {, }
$$


donde

$K^{* *}=$ Número de variables predeterminadas o exógenas excluidas en la $g^{m}$ ecuación.

$M^{* *}$. Número de variables dependientes o exógenas incluidas en la $g^{m}$ ecuación.

Con esto, se elaborará un modelo univariado de series de tiempo de la inflación como instrumento del tipo de cambio, con las características usuales:

$$
\left(1-B^{n}\right)\left(1-B^{n}\right) L P_{t}=\left(1-B^{1}+B^{2}+\cdots+B^{n}\right)\left(1-B^{n}\right) A_{t}
$$

donde $A_{t}$ es la parte estócastica.

En cuanto al tipo de cambio, su relación de comportamiento se basa en la especificación de la Hipótesis Abierta de Fisher. Se usará para tal efecto a la tasa externa (treasury bills a 3 meses $(T B)$ ) y como variables exógenas a la tasa de interés doméstica (TIIE), al ingreso interno (índice de la producción industrial $(Y)$ ), la oferta monetaria $\left(M 3\right.$ ó $M 4\left(M^{S}\right)$ ) y la Inflación Esperada $\left(D L P^{e}\right)$. Se utiliza también una variable dicotómica $(D U M T C)$ que captura los efectos inducidos sobre el tipo de cambio. ${ }^{9}$.

$$
(1-B) T C=\beta_{\circ}\left(M^{S}\right)^{\beta_{1}} Y^{\beta_{2}}\left(D L P^{e}\right)^{\beta_{3}} T B^{\beta_{4}} D U M T C^{\beta_{5}} U_{t} .
$$

En relación con la inflación se especifica un modelo en primeras diferencias tradicional, en donde se incluyen factores como inflación importada (precios externos al mayoreo en USA ( $P E M)$ ), el tipo de cambio nominal pesos por dólar esperaito $\left(T C^{e}\right)$, inflación de costos (salario mínimo nominal $\left(W_{n}\right)$ ) y los precios de bienes públicos $(P E P)$. También se incluyen en la ecuación al ingreso interno $(Y)$, a la oferta monetaria $\left(M^{s}\right)^{10}$ y a una variable dicotómica (DUMP). Finalmente, es necesario mencionar que esta especificación podría resultar en un proceso de raíces unitarias (para mayor detalle véase el apéndice B).

$$
\left(1-B^{2}\right) P=\gamma_{o}\left(T C^{e}\right)^{\gamma_{1}} W n^{\gamma_{2}} P E M^{\gamma_{3}} Y^{\gamma_{4}} P E P^{\gamma_{5}} M^{s \gamma_{6}} D U M P^{\gamma_{7}} V_{t} .
$$

Respecto a las tasas de interés en los diferentes plazos, se utilizó la especificación propuesta en (5). Despejando la tasa de interés doméstica (CETES a 28, 91, 182 y 364 días; $\left.C T P_{28}, C T P_{91}, C T P_{182}, C T P_{364}\right)$ ) se obtiene la especificación propuesta, la cual se generaliza para la estimación de la EITI. Cabe señalar, que adicionalmente se incluyeron variables como $S T^{u s a}$ que es un spread entre el bono corto y el bono largo en USA, la variable DITE que mide el diferencial entre el bono corto en USA y su similar en México, $S T^{P 3 M}$ que representa el spread entre los BCC a plazos de 3 y 6 meses. Por último, se incluyó a la variable $S T^{P 1 A}$ que expresa la dispersión entre los bonos cupón cero de 1 y 12 meses.

$$
\begin{aligned}
(1-B) C T P_{28}= & \varphi_{o}\left(T C^{e}\right)^{\varphi_{1}}\left(S T^{u s a}\right)^{\varphi_{2}} T I I E^{\varphi_{3}}\left(M^{S}\right)^{\varphi_{4}}\left(D L P^{e}\right)^{\varphi_{5}} Y^{\varphi_{6}} \times \\
& D U M C T^{\varphi_{7}} N_{t_{1}} .
\end{aligned}
$$

9 Adaptado del trabajo de Tapia (1990).

10 Véase Tapia (1992). 


$$
\begin{aligned}
(1-B) C T P_{91}= & \theta_{o}\left(T C^{e}\right)^{\theta_{1}} D I T E^{\theta_{2}} T I I E^{\theta_{3}}\left(M^{S}\right)^{\theta_{4}}\left(D L P^{e}\right)^{\theta_{5}} Y^{\theta_{6}} \times \\
& D U M C T^{\theta_{7}} N_{t_{2}} \cdot \\
(1-B) C T P_{182}= & \xi_{o}\left(T C^{e}\right)^{\xi_{1}}\left(S T^{P 3 M}\right)^{\xi_{2}} T I I E^{\xi_{3}}\left(M^{S}\right)^{\xi_{4}}\left(D L P^{e}\right)^{\xi_{5}} Y^{\xi_{6}} \times \\
& \left(S T^{U S A}\right)^{\xi_{7}} D U M C T^{\xi_{8}} N_{t_{3}} \cdot \\
(1-B) C T P_{364}= & \psi_{o}\left(T C^{e}\right)^{\psi_{1}}\left(S T^{P 1 A}\right)^{\psi_{2}} T I I E^{\psi_{3}}\left(M^{S}\right)^{\psi_{4}}\left(D L P^{e}\right)^{\psi_{5}} Y^{\psi_{6}} \times \\
& \left(S T^{U S A}\right)^{\psi_{7}} D U M C T^{\psi_{8}} N_{t_{4} \cdot} .
\end{aligned}
$$

\section{Estimación y resultados}

\subsection{Estimación}

Se utilizó un período muestral de corte mensual desde enero de 1993 a marzo de 1999. Cabe resaltar que en esta etapa se presentaron diversas crisis financieras, una interna en 1995 y otras externas en 1997 y 1998, como son la de Asia Pacífico, Rusia y Brasil. En el sistema propuesto de ecuaciones simultáneas (ES) - Riesgo de Mercado - y el sistema de ecuaciones similares pero no relacionadas (SUR) - EITI -, la especificación es doble logarítmica con el objetivo de interpretar los coeficientes estimados como elasticidades.

\subsection{Riesgo de mercado}

Para la estimación del riesgo de mercado (el tipo de cambio nominal, la inflación y la tasa de interés de los certificados de la tesorería a plazo de 28 días) se obtuvieron los siguientes resultados:

\subsubsection{Tipo de cambio nominal}

En la ecuación del tipo de cambio, se utilizó como variable instrumental de inflación un modelo univariado de series de tiempo, con el propósito de purgar en esta ecuación los choques estocásticos de la inflación. El instrumento mencionado cuenta con las siguientes características: ${ }^{11}$

$$
\begin{gathered}
\left(1-0.59 B^{1^{*}}\right)(1-B) L P_{t}=\left(1-\left(0.40 B^{1^{*}}+0.19 B^{2^{* *}}+0.40 B^{3^{*}}\right)\right)\left(1-0.27 B^{12^{*}}\right) A_{t} \\
(0.119) \\
(0.118) \quad(0.123) \quad(0.112)
\end{gathered}
$$

11 Entre paréntesis se indican las desviaciones estándar de los coeficientes estimados. Un asterisco indica una significación al nivel de $5.0 \% \mathrm{y}$ dos asteriscos indican que no es significativamente diferente de cero. 
En relación con el tipo de cambio se consideró el uso de una regresión con las siguientes consideraciones:

$$
\begin{gathered}
\left(1-0.838 B^{*}\right) T C=-1.257^{*}+0.141 M 3^{*}-0.104 Y+2.055\left(D L P^{e}\right)^{*}+0.064 T B^{*} \\
(0.039) \quad \begin{array}{cccc}
(0.365) & (0.049) & (0.113) & (0.574) \\
+ & 0.044 D U M T C^{*}
\end{array} \\
(0.004) \\
\bar{R}^{2}=0.994, h=1.54, S E R=0.031, J B=1.297, Q_{24}=31.290 \\
\text { Figura 4. Tipo de Cambio. }
\end{gathered}
$$

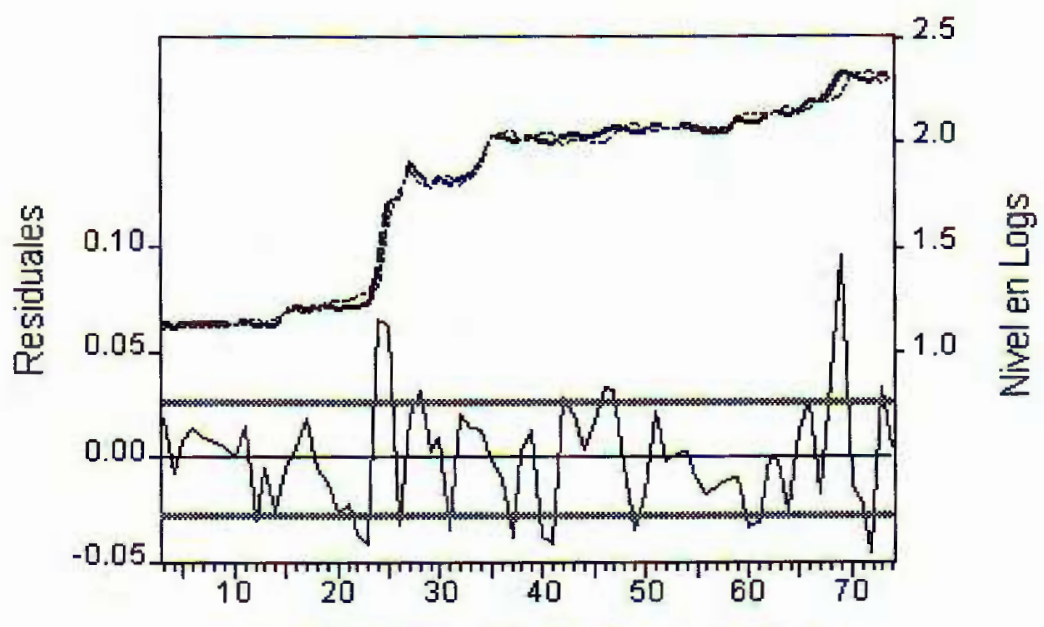

-Residual —-Actual -..-.-Fitted

\subsubsection{Inflación}

Respecto a la determinación de la inflación, el modelo especificado se presenta en primeras diferencias, a fin de establecer el ritmo en que crecen las variables tanto dependientes como independientes. La propuesta es la siguiente: 


$$
\begin{aligned}
& \left(1-0.57 B^{*}\right) D L P=0.004^{*}+0.041\left(D T C^{e}\right)^{*}+0.087 D W n^{*}+0.132 D L P E M \\
& (0.0007)(0.007) \\
& +0.047 D M 4^{*}-0.017 D Y^{*}+0.121 D L P E P^{*}+0.002 D U M P^{*} . \\
& \bar{R}^{2}=0.945, h=0.14, S E R=0.003, J B=1.466, Q_{24}=14.493 .
\end{aligned}
$$

Figura 5. Inflación.

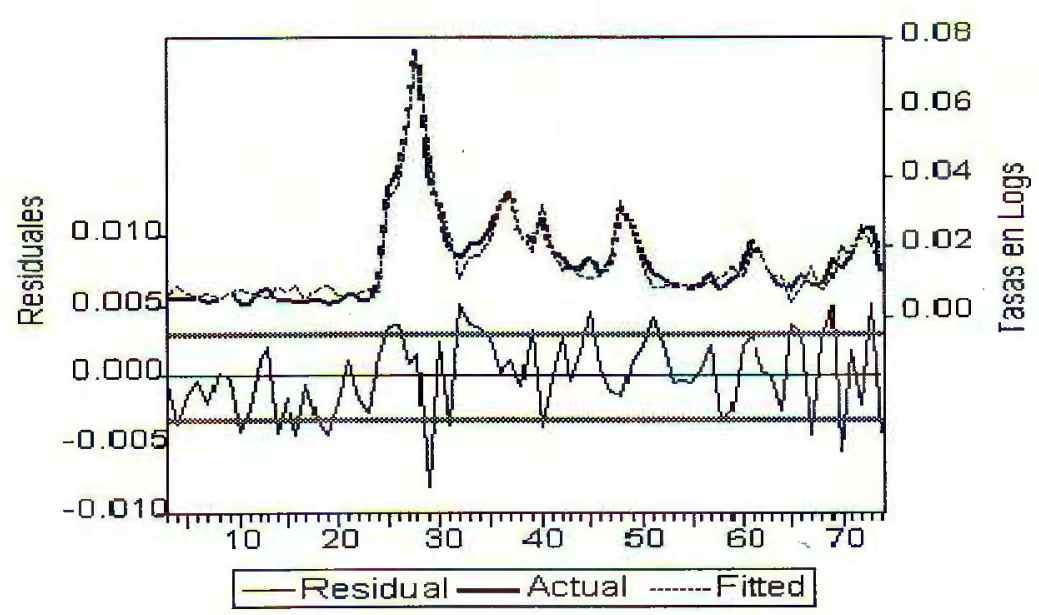

\subsubsection{CETES a 28 días}

En la presente ecuación se usó el promedio mensual y contiene como instrumentos tanto el tipo de cambio como la inflación estimados en las ecuaciones anteriores; esto con el fin de separar la posible correlación serial entre los errores de las diferentes regresiones.

$$
\begin{aligned}
& \left(1-0.277 B^{*}\right) C T P=3.970^{*}+0.611\left(T C^{e}\right)^{*}+0.120 S T^{U S A}+0.551 T I I E^{*} \\
& \text { (0.036) } \\
& \text { (0.940) (0.086) } \\
& \text { (0.068) } \\
& -0.536 M 4^{*}+3.769\left(D L P^{e}\right)^{*}+0.573 Y^{*}+0.22 D U M C T^{*} . \\
& (0.099) \quad(0.849) \\
& \text { (0.191) (0.002) }
\end{aligned}
$$

$$
\bar{R}^{2}=0.988, h=0.09, S E R=0.510, J B=0.155, Q_{24}=33.020 .
$$

Desde luego que en la determinación de los riesgos de mercado se elaboraron, para identificar al sistema, las condiciones de orden y de rango a partir de la especificación:

$$
K^{* *}>M^{* *}-1 \text {. }
$$


Por otro lado, a fin de corroborar la ortogonalidad de la parte estocástica del sistema, se presenta la matriz de correlación de errores del mismo. Como se puede apreciar los resultados son satisfactorios.

\begin{tabular}{l|c|c|c|}
\multicolumn{1}{c}{} & \multicolumn{1}{c}{ Mep } & \multicolumn{1}{c}{ Metc } & Mect \\
\cline { 2 - 4 } Mep & 1.000 & 0.306 & 0.116 \\
\cline { 2 - 4 } Metc & 0.306 & 1.000 & 0.236 \\
\cline { 2 - 4 } Mect & 0.116 & 0.236 & 1.000 \\
\cline { 2 - 4 } & &
\end{tabular}

\subsection{Estructura intertemporal de las tasas de interés}

La condicionante para la estimación de la estructura se basa en los modelos SUR. Sin embargo, los supuestos de errores esféricos no son aplicables y por lo tanto es menester corregirlos a partir de un sistema generalizado con ponderadores de los componentes estocásticos. A continuación se describen los resultados:

CETES a 28 días

$$
\begin{aligned}
& \left(1-0.277 B^{*}\right) C T P_{28}=3.970^{*}+0.611 T C^{e^{*}}+0.120 S T^{U S A}+0.551 T I E^{*} \\
& \begin{array}{lllll}
(0.036) & (0.940) & (0.086) & (0.068) & (0.031)
\end{array} \\
& -0.536 M 4^{*}+3.769 D L P^{e^{*}}+0.573 Y^{*}+0.22 D U M C T_{28^{*}} . \\
& \begin{array}{llll}
(0.099) & (0.849) & (0.191) & (0.002)
\end{array} \\
& \bar{R}^{2}=0.988, h=0.09, S E R=0.051, J B=0.155, Q_{24}=33.020 .
\end{aligned}
$$

Figura 6. Cetes a 28 días.

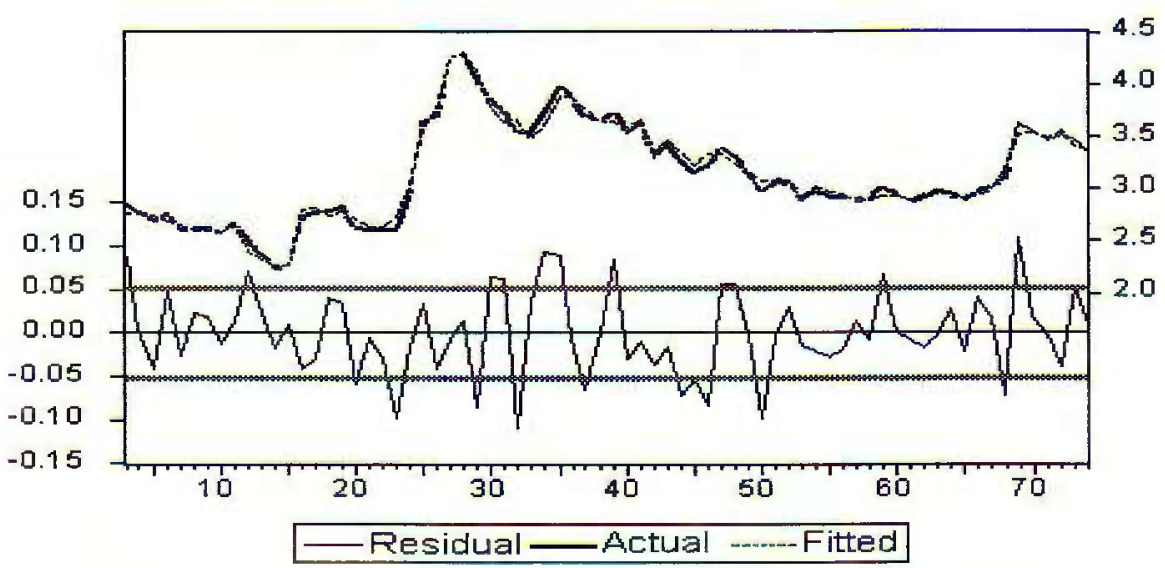


CETES a 91 días

$$
\begin{aligned}
& \left(1-0.229 B^{*}\right) C T P_{91}=2.529^{*}+0.413 T C^{e^{*}}-0.423 M 1^{*}+1.342 D L P^{e^{*}}+0.55 Y^{*} \\
& (0.027) \\
& \text { (0.435) (0.036) } \\
& (0.050) \\
& \text { (0.525) } \\
& +0.405 T I E^{*}+0.237 D I T E^{*}+0.013 D U M C T_{91^{*}} \text {. } \\
& \bar{R}^{2}=0.995, h=1.39, S E R=0.031, J B=0.826, Q_{24}=23.513 \text {. }
\end{aligned}
$$

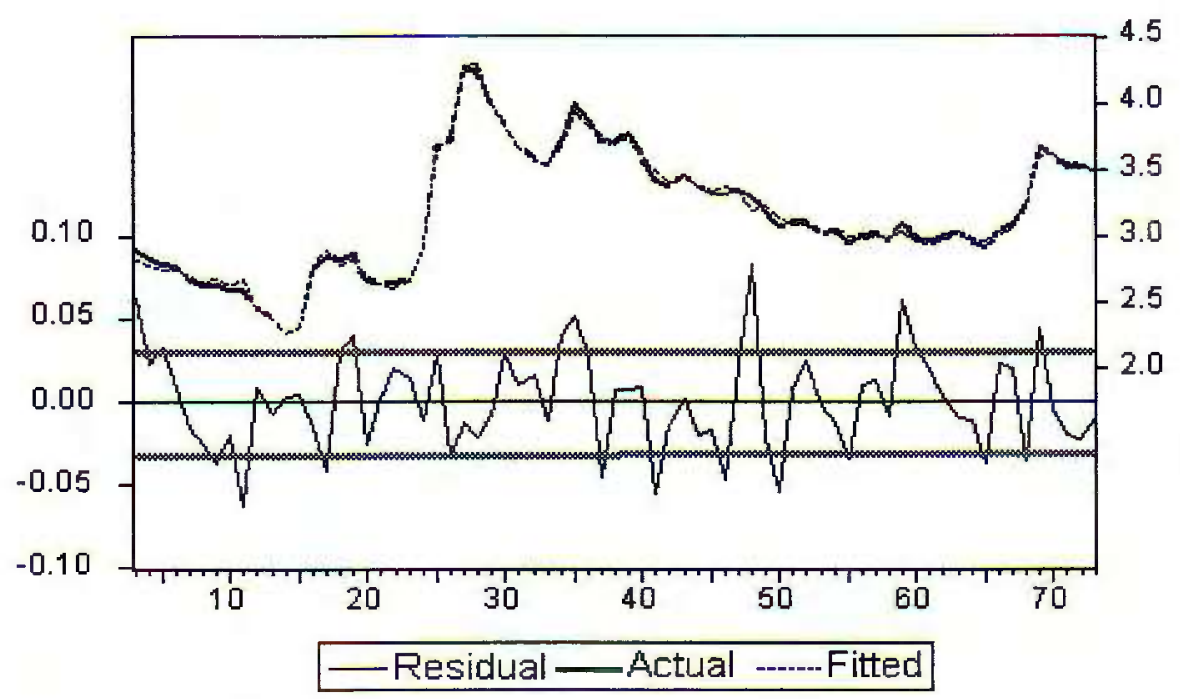

CETES a 182 días

$C T P_{182}=5.840^{*}+0.806 T C^{e^{*}}-0.625 M 1^{*}+3.966 D L P^{e^{*}}+0.441 T I E^{*}+0.420 Y^{*}$
(1.279) (0.097)
$(0.101)$
(0.931)
$(0.042)$
$-0.201 S T^{P 3 M}-0.048 S T^{U S A}+0.014 D U M C T 182^{*}+0.815 A R(1)^{*}$.
(0.158)
(0.077)
(0.001)
(0.073)

$\bar{R}^{2}=0.989, S E R=0.0463, R I A R=0.820, J B=1.308, Q_{24}=10.764$. 
Figura 8. Cetes a 182 días.

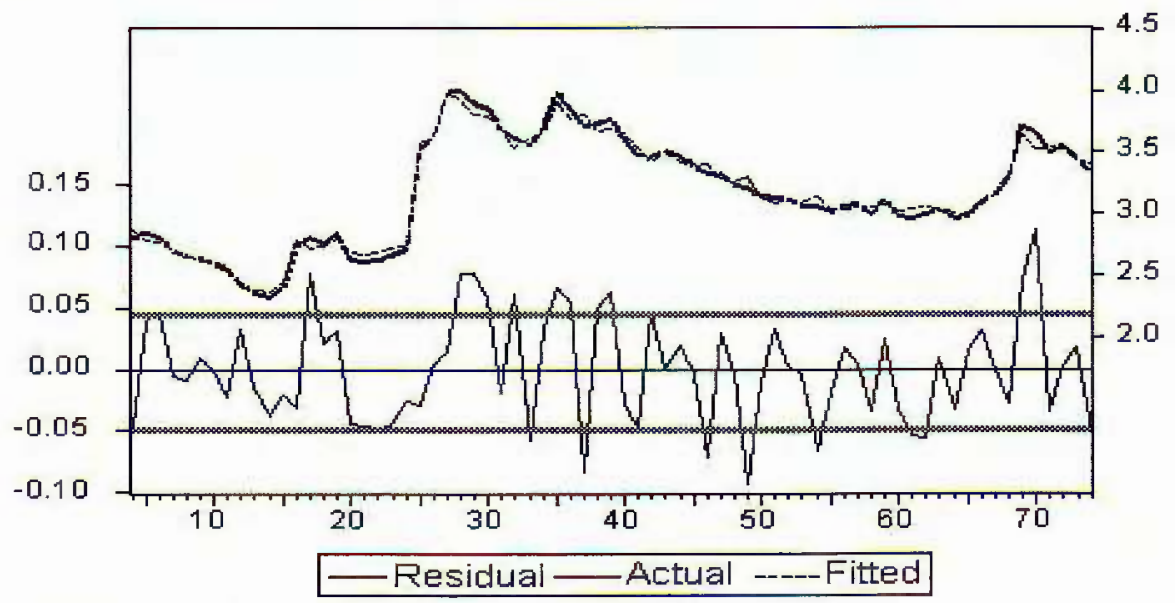

CETES a 364 días

$C T P_{364}=12.13^{*}+1.558 T C^{e^{*}}-1.235 M 4^{*}+2.64 D L P^{e^{*}}+0.43 S T^{P 1 A^{*}}+0.809 Y^{*}$

$$
\begin{aligned}
& \begin{array}{lllll}
(1.304) & (0.095) & (0.106) & (0.740) & (0.051)
\end{array} \\
& +0.413 T I I E^{*}+0.128 S T^{U S A *}+0.017 D U M C T 364^{*}+0.787 A R(1)^{*} \text {. } \\
& \begin{array}{llll}
(0.030) & (0.062) & (0.0009)
\end{array}
\end{aligned}
$$$$
\bar{R}^{2}=0.992, S E=0.038, R I A R=0.790, J B=2.100, Q_{24}=23.455 .
$$

Figura 9. Cetes a 364 días.

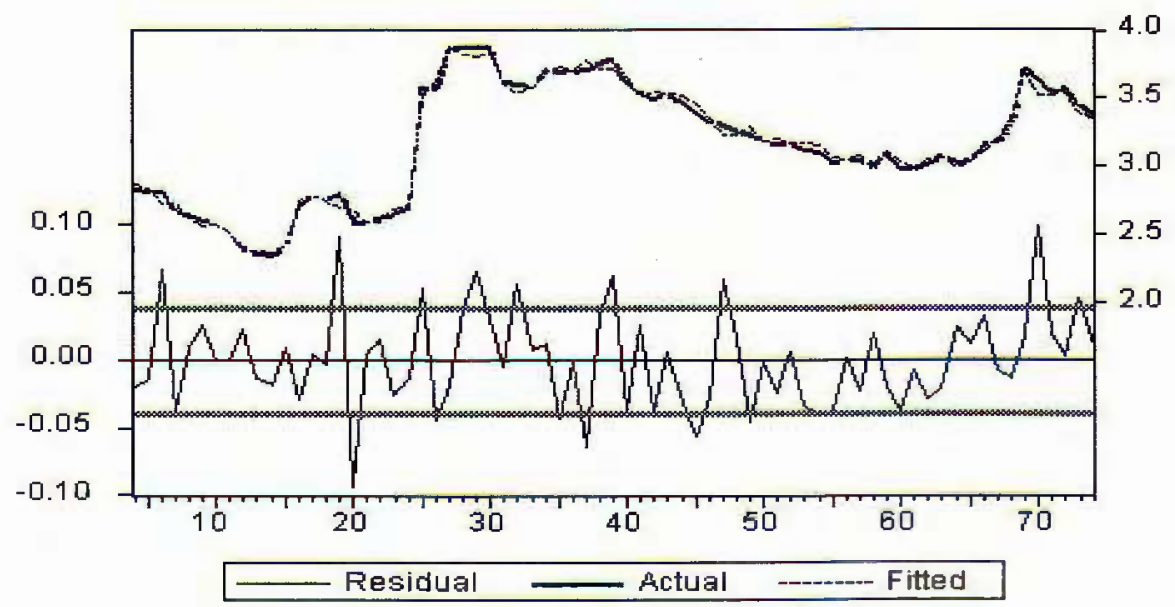


De hecho, para cerciorarnos de la ortogonalidad del sistema se presenta la matriz de correlaciones de la parte estocástica. Como se puede observar, los resultados son aceptables:

\begin{tabular}{c|c|c|c|c|} 
& \multicolumn{1}{c}{$\mathrm{Me}_{28}$} & $\mathrm{Me}_{91}$ & $\mathrm{Me}_{182}$ & $\mathrm{Me}_{364}$ \\
\cline { 2 - 5 } $\mathrm{Me}_{28}$ & 1.000 & 0.538 & 0.308 & 0.372 \\
\cline { 2 - 5 } $\mathrm{Me}_{91}$ & 0.538 & 1.000 & 0.248 & 0.209 \\
\cline { 2 - 5 } $\mathrm{Me}_{182}$ & 0.308 & 0.248 & 1.000 & 0.386 \\
\cline { 2 - 5 } $\mathrm{Me}_{364}$ & 0.372 & 0.209 & 0.386 & 1.000 \\
\cline { 2 - 5 } & &
\end{tabular}

Con el fin de llevar a cabo una prueba robusta sobre si los errores de los 2 sistemas son homoscedásticos, en otras palabras esféricos, se anexa la matriz de errores conjunta. En este caso, también podemos observar que los resultados que arroja el sistema son tolerables:

\begin{tabular}{|c|c|c|c|c|c|c|}
\hline & Mep & Metc & $\mathrm{Me}_{28}$ & $\mathrm{Me}_{91}$ & $\mathrm{Me}_{182}$ & $\mathrm{Me}_{364}$ \\
\hline Mep & 1.00 & 0.31 & 0.13 & -0.02 & 0.16 & -0.08 \\
\hline Metc & 0.31 & 1.00 & 0.23 & 0.13 & 0.38 & 0.29 \\
\hline $\mathrm{Me}_{28}$ & 0.13 & 0.23 & 1.00 & 0.53 & 0.30 & 0.37 \\
\hline $\mathrm{Me}_{91}$ & -0.02 & 0.13 & 0.53 & 1.00 & 0.24 & 0.20 \\
\hline $\mathrm{Me}_{182}$ & 0.16 & 0.38 & 0.30 & 0.24 & 1.00 & 0.38 \\
\hline $\mathrm{Me}_{36}$ & -0.08 & 0.29 & 0.37 & 0.20 & 0.38 & 1.00 \\
\hline
\end{tabular}

\subsection{Resultados}

La variación explicada, $\bar{R}^{2}$, de los modelos fue alta, mientras que las pruebas de Durbin, $h$, muestran que no existe correlación serial de primer orden. El error estándar de las estimaciones $(S E R$ ) fue aceptable, los residuales evidenciaron ser ruido blanco por el valor reportado en el estadístico Ljung-Box $(Q)$ y el estadístico Jarque Bera $(J B)$ resultó satisfactorio. Las Raíces Invertidas Autorregresivas ( $R I A R$ ), que evidencian la estabilidad de las estimaciones, también resultaron satisfactorias.

Las variables dicotómicas, empleadas para señalar los eventos de crisis financieras de México, Asia Pacífico, Rusia y Brasil, resultaron significativas en todos los casos. La ortogonalidad resultante, tanto del sistema de Riesgo de Mercado $(E S)$, de la Estructura Intertemporal de las Tasas de Interés $(S U R)$ y del Sistema Total, muestran que la bondad del ajuste es satisfactoria.

\subsubsection{Tipo de cambio}

Con relación a la serie de tiempo de precios utilizada en la ecuación del tipo de cambio nominal, cabe señalar que en la parte de promedios móviles regulares, la suma de sus coeficientes son estrictamente menores a la unidad y para el caso del segundo coeficiente su significación estadística no difiere de cero. El estadístico Ljung-Box indica que los errores son ruido blanco.

La ecuación del tipo de cambio muestra los signos correctos. En este sentido,

$$
\partial T C / \partial M^{s}>0, \quad \partial T C / \partial D L P^{e}>0, \quad \partial T C / \partial T B>0,
$$


causan una depreciación nominal, mientras que

$$
\partial T C / \partial Y<0
$$

coincide con lo predicho por la teoría cuantitativa del dinero. En otras palabras, un aumento del ingreso provoca un incremento en la demanda de dinero, lo que ocasiona a su vez la elevación de la tasas de interés domestica y, por lo tanto, propicia una entrada de capitales.

\subsubsection{Inflación ${ }^{12}$}

La dinámica de la inflación responde directamente a los factores externos, como la inflación importada, así como a factores de costos y de precios públicos. ${ }^{13} \mathrm{~A}$ partir de esto, se obtuvieron los siguientes signos:

$\partial D L P / \partial P E M>0, \partial D L P / \partial T C^{e}>0, \partial D L P / \partial W n>0$ y $\partial T C / \partial P E P<0$.

Por otra parte, la expansión de la actividad económica reduce el crecimiento de precios, ya que al aumentar el ingreso aumenta la demanda de dinero y se incrementa la tasa de interés doméstica, reduciendo el nivel del gasto en la economía. Finalmente, la expansión monetaria incrementa los precios.

$$
\partial D L P / \partial Y<0, \quad \partial D L P / \partial M^{s}>0
$$

\subsubsection{Estructura intertemporal de tasas de interés}

Respecto a las tasas de interés a plazo de 28 días, éstas aumentan al incrementarse el tipo de cambio esperado, $T C^{e}$, y la inflación esperada, $D L P^{e}$. Lo anterior, con el fin de mantener el inward arbitraje y las tasas reales.

$$
\partial C T_{28} / \partial T C^{e}>0, \quad \partial C T_{28} / \partial D L P^{e}>0
$$

Asimismo, al aumentar la expansión productiva se incrementa la demanda de dinero, disminuyendo el precio de los bonos y subiendo la tasa de interés. Un incremento en la TIIE o en la demanda de crédito, genera una caida en la demanda de bonos y un aumento en la tasa de interés. Finalmente, al aumentar el spread entre el bono corto y largo en EE.UU. ( $\left.S T^{U S A}\right)$, tomando a éste como una medida de volatilidad, se eleva la tasa de interés por la incertidumbre en el mercado. En otras palabras:

$$
\partial C T_{28} / \partial Y>0, \quad \partial C T_{28} / \partial T I I E>0, \quad \partial C T_{28} / \partial S T^{U S A}>0
$$

12 La representación de la dinámica inflacionaria requiere de un tratamiento adicional dada la expresión en diferencias, para mayor detalle véase el apéndice $B$.

13 Se considera que la expansión monetaria, primero, incrementa los recursis ociosos y posteriormente los precios. 
Para el caso del efecto riqueza, al incrementarse el agregado monetario, $M^{\boldsymbol{s}}$, aumenta la demanda por bonos y cae la tasa de interés. ${ }^{14}$ Por tal motivo,

$$
\partial C T_{28} / \partial M^{s}<0 .
$$

Para el plazo de 91 días, los efectos del tipo de cambio esperado, $T C^{e}$, la inflación esperada, $D L P^{e}$, el ingreso, $Y$, la $T I I E$ y el agregado monetario restringido, $M^{s}$, son los mismos, aunque con magnitudes diferentes. Adicionalmente, se añade una variable de volatilidad, que se mide a través del diferencial de tasas entre el bono corto en USA y su similar en México, DITE. Con esta nueva variable se puede concluir que, entre mayor sea el diferencial es necesario incrementar las tasas internas para evitar la salida de capitales.

$$
\partial C T 9_{91} / \partial D I T E>0 .
$$

De la misma forma, para el plazo de 182 días, los signos del tipo de cambio esperado, $T C^{e}$, la inflación esperada, $D L P^{e}$, el ingreso, $Y$, la $T I I E$, el agregado monetario restringido, $M^{\boldsymbol{s}}$, y el diferencial entre el bono corto y largo en EE.UU. $S T^{U S A}$, son iguales a los de los plazos antes descritos, aunque con diferentes impactos. ${ }^{15}$ En este caso, se incluye una variable que mide el diferencial entre los $\mathrm{BCC}$ de 3 y 6 meses $S T^{P 3 M}$ como un proxy de la pendiente de la EITI en el período contemporáneo. En este sentido, a medida que éste aumente, la variable independiente tenderá a estabilizarse contrarrestando el efecto inicial.

$$
\partial C T_{182} / \partial S T^{P 3 M}<0 .
$$

En cuanto al plazo de un año, los signos observados son los esperados. La variable $S T^{P 1 A}$, la cual mide el diferencial entre los $B C C$ entre 1 y 12 mes, presenta un signo positivo. Esto quiere decir que a medida que el diferencial aumente, la dispersión entre los plazos de la EITI en el periodo contemporaneo, tenderá a estabilizarse.

$$
\partial C T_{364} / \partial S T^{P 1 a}>0 .
$$

Es importante señalar que durante el período de estudio se presentaron diversos choques internos y externos que afectaron a las variables económicas fundamentales. Entre estos choques están la crisis mexicana de principios de 1995, las acontecidas en el sureste asiático a mediados de 1997 y la debacle rusa precipitada en el año de 1998. Por lo anterior, se elaboraron pruebas de cambio estructural de Chow y $C U S U M^{16}$ con el fin de corroborar la estabilidad de los sistemas planteados.

A partir de la siguiente tabla, podemos concluir lo siguiente: para el caso del Tipo de Cambio, el registro del choque estructural se llevó a cabo esencielmente en la crisis rusa y brasileña. En cuanto a la inflación, el cambio estructural se observa en la crisis mexicana. Finalmente, con relación a las

\footnotetext{
14 La consideración explícita que se hace es que los bonos gubernamentales son outsidebonds y el dinero es outsidemoney.

15 Esta representación es similar a la utilizada por Shiller and McCulloch (1991).

16 Para mayor detalle véase el Apéndice C.
} 
tasas de interés el punto de inflexión de la EITI se registró en la de plazo de 3 meses para la crisis mexicana; mientras que para las crisis del sureste asiático, la rusa y brasileña se concentra en la de plazo de un año. No obstante, las pruebas de $C U S U M$ arrojaron una estabilidad en la suma acumulada de residuales, como se observa en las siguientes gráficas.

\begin{tabular}{|c|c|c|c|}
\hline \multicolumn{4}{|c|}{ Pruebas de Cambio estructural } \\
\hline & Crisis Mexicana & $\begin{array}{c}\text { Crisis de Sureste } \\
\text { Asiático }\end{array}$ & Crisis Rusa y Brasileña \\
\hline Tipo de Cambio & 2.26 & 3.11 & $3.71^{*}$ \\
\hline Inflación & $3.34^{*}$ & 1.33 & 1.45 \\
\hline Tasas de interés & & & 1.49 \\
\hline Cete a 1 mes & 1.75 & 1.07 & 0.97 \\
\hline Cetes a 3 meses & $3.93^{*}$ & 1.49 & 1.29 \\
\hline Cetes a 6 meses & 0.02 & 0.82 & 2.6 \\
\hline Cetes a 1 año & 1.49 & 2.56 & \\
\hline
\end{tabular}

* significa que se rechaza la hipótesis nula al $1 \%$ de significancia. Pruebas $F_{t-2 k}^{k}$.

Figura 10. Inflación.

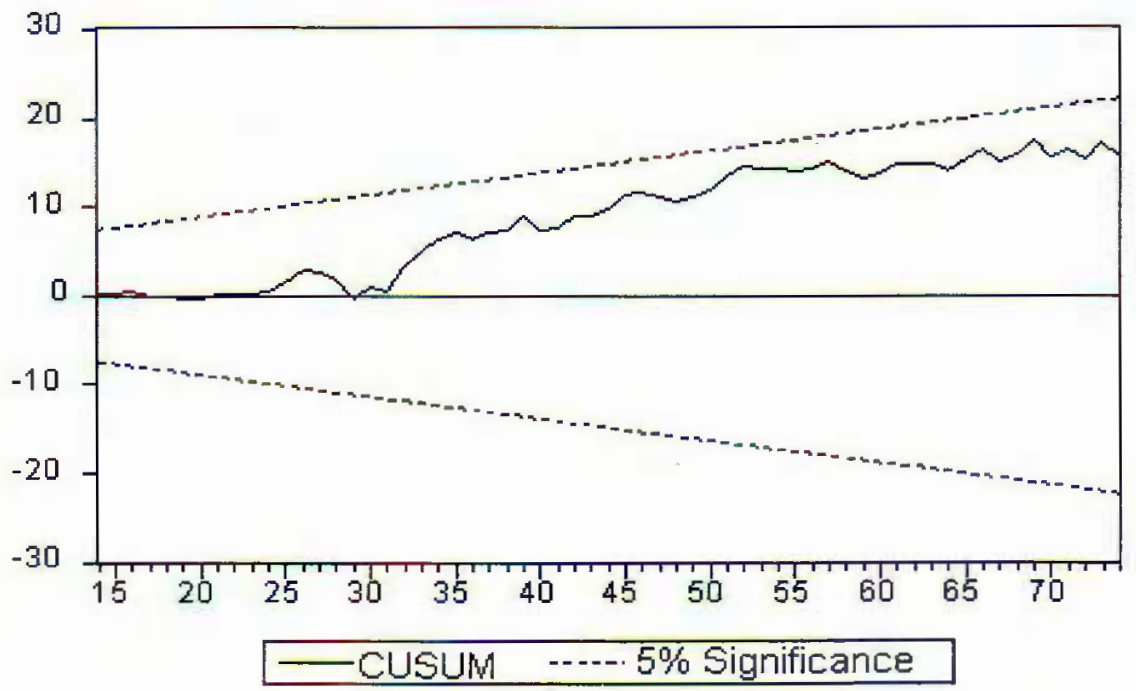


Revista Mexicana de Economía y Finanzas, Vol. 2, No. 1, (2003), pp. 49-80

Figura 11. Tipo de Cambio.

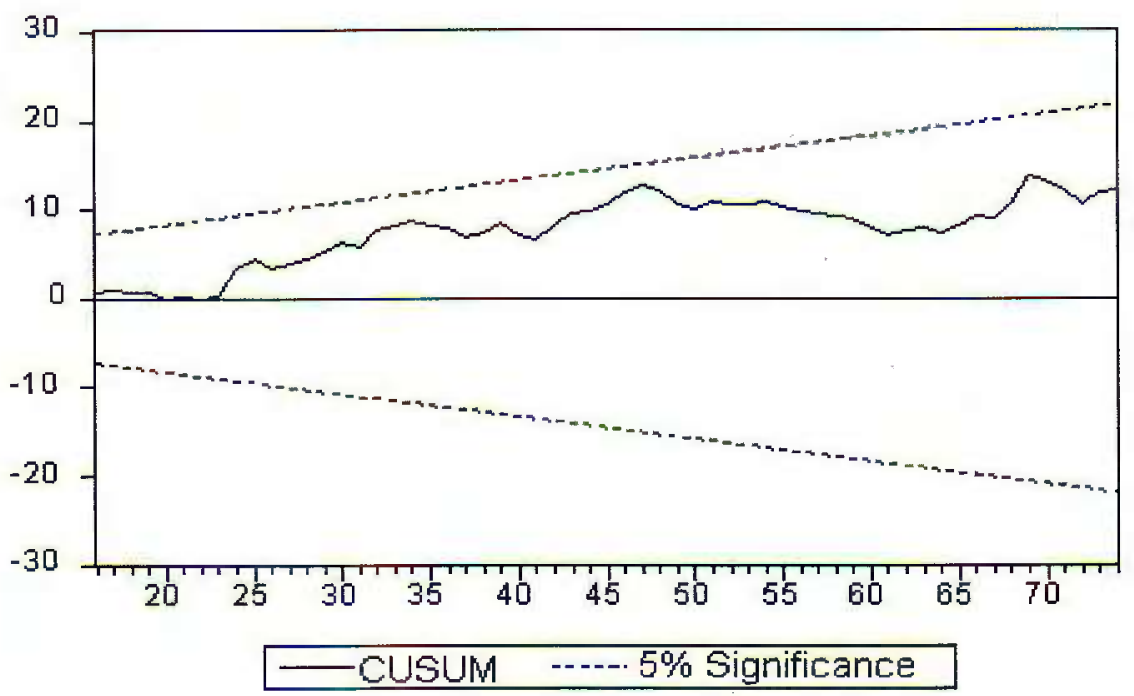

Figura 12. Cetes a 28 días.




Figura 13. Cetes a 91 días.

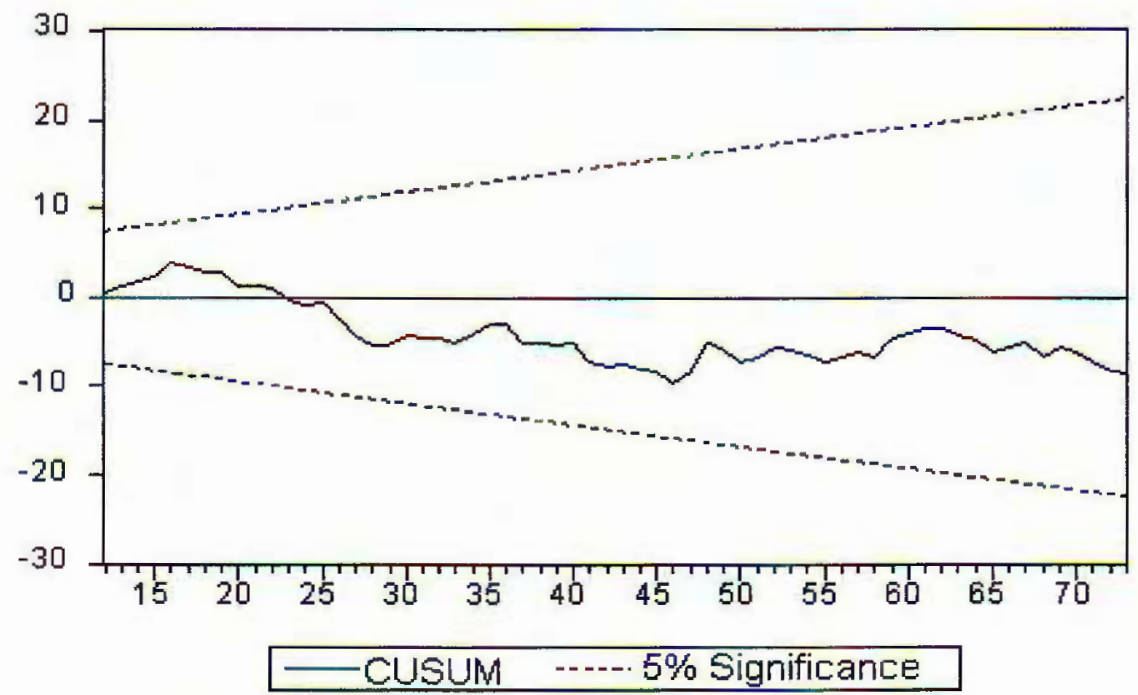

\section{Conclusiones}

Los resultados obtenidos contribuyen a una mejor comprensión del mercado de dinero y a un mayor conocimiento de los riesgos de mercado desde una perspectiva de expectativas racionales. A partir de las diferentes velocidades de ajuste de los sectores económicos (cambiario, de dinero y de bienes) se puede definir un patrón de comportamiento producido por un choque de información, distinguiendo no sólo la dirección sino la magnitud de los ajustes. Las representaciones finales y las matrices de correlación de errores arrojan los efectos cruzados entre las ecuaciones del sistema de riesgo de mercado y la forma en que estos choques estocásticos se pueden transmitir entre las diversas ecuaciones.

La importancia de este tipo de ejercicio es que con base en variables del mercado, se hace una mejor y más robusta interpretación de la realidad. De tal forma que se espera que las señales proporcionadas por la autoridad tengan una mejor recepción por parte de todos los participantes del mercado y que estos hagan una lectura más clara de éstas. ${ }^{17}$ Asimismo, el sistema global puede ser un instrumento para evaluar efectos inducidos de política monetaria o cambiaria

17 En contraste con otro tipo de modelos para evaluar los riesgos de mercado, como lo serían los modelos de series univariadas o multivariadas de tiempo, basados en los dos primeros momentos de la función generatriz, resultan parciales en cuanto al funcionamiento del mercado en su conjunto. Adicionalmente, la generación de números aleatorios normales estándar a través de procesos de Wiener, también producen información aislada de la representación del mercado. 
con el fin de alcanzar algún objetivo. Esto a través del análisis de la reacción en los riesgos de mercado ante diferentes alteraciones.

Para mantener una paridad cambiaria estable es requisito fundamental que la autoridad monetaria pueda contener la inflación esperada, tenga una regla monetaria prudente y que pueda reaccionar ante los movimientos del mercado de bonos norteamericano. En cuanto a la política inflacionaria, además de la contención monetaria, es preciso mantener en línea las expectativas cambiarias. Cabe señalar que una política de precios relativos prudente del sector público apoya al combate de la inflación. Sin embargo, el proceso de apertura de la economía mexicana se traduce en una mayor vulnerabilidad, a través de la inflación importada, y una política salarial holgada se transmitirá irremediablemente en presiones inflacionarias.

La estructura intertemporal permite distinguir la sensibilidad de las tasas de interés a diferentes plazos a partir del cambio de algunas variables. Dada la experiencia del período observado, la estabilidad de EITI fue aceptable, aunque en general hubo diversos tipos de estructura, es decir, con pendiente positiva (4 años), negativa (2 años) y plana (trimestral). No obstante, las relaciones de comportamiento planteadas se preservaron.

Dentro de la EITI los plazos cortos de 28 y 91 días presentan similitudes que merecen comentarse pues ambos son los más demandados y son sobre los que existen las mayores posiciones en riesgo. La representación propuesta es consistente con la del equilibrio al final de período, permitiendo con ello el ajuste consistente de flujos y stocks. Para este tipo de instrumentos, la mejor política para reducir la tasa de interés es sin duda la contención de la inflación y del tipo de cambio esperado. El efecto riqueza tiene un impacto decisivo de acuerdo con el punto de vista keynesiano, ya que éste mide la expansión de la liquidez a través de su efecto colateral en la disminución de tasas, al menos en el corto plazo. Es también importante la sensibilidad de las tasas de interés ante el entorno financiero externo, especialmente, frente a los diferenciales que existen entre las tasas internas y externas.

En cuanto a las tasas de largo plazo, éstas son sensibles a los diferenciales entre las tasas externas y a la convexidad de la EITI. La ortogonalidad del sistema, tanto de los riesgos de mercado como los de la EITI, permiten ver los efectos cruzados de los residuales. Con esto podemos llevar a cabo un mejor análisis de la interdependencia estocástica del sistema y corroborar que es robusto en términos de las variables económico-financieras. Desde luego que este tipo de planteamientos arrojará resultados mas o menos eficientes de acuerdo al tipo de información que se posea y a los fines que se persigan.

La estabilidad de la EITI depende en gran medida de la existencia de un entorno macroeconómico favorable, asi como de la coordinación de los principales actores en el mercado, en cuanto a la lectura y análisis de la información existente. Finalmente, la asimetría de los choques internos y externos en la economía, se evidenciaron a través de las pruebas de cambio estructural. En la crisis mexicana de 1995, el impacto fue directamente sobre los niveles de inflación y en las tasas de interés a 91 días, lo cual se vió reflejado en la pendiente de la EITI. En lo que se refiere a las crisis externas, hubo un impacto mayor sobre el mercado cambiario y en las tasas de interés a plazo de 1 año. 


\section{Apéndice $\mathbf{A}$}

Tradicionalmente, la determinación de las tasas de interés se sustenta en la teoría de los fondos prestables o en la preferencia por la liquidez, ambos bajo un enfoque de equilibrio parcial en donde ésta se establece a partir del mercado de bonos, en el primer caso, y en el de dinero, en el segundo. ${ }^{18}$

\section{A.1 Fondos prestables}

Esta teoría presupone que la tasa de interés de los bonos es inversamente proporcional a su precio.

$$
P=C / r,
$$

donde $C$ es el cupón y $r$ es la tasa de interés. Las características esenciales de esta teoría son: la oferta planeada de bonos es igual al nivel planeado de inversión por parte de las empresas,

$$
B^{s}=I .
$$

Por otra parte, la demanda planeada de bonos $\left(B^{d}\right)$ iguala al ahorro planeado de las unidades de consumo existentes dentro de la economía $(S)$. Cabe hacer notar que la inversión y el ahorro son flujos, los cuales se relacionan con los flujos de oferta y demanda de bonos. Se supone que la tasa de interés está determinada en el mercado de bonos, donde la condición de equilibrio es:

$$
B^{d}=B^{s} \text {. }
$$

O en su caso:

$$
S=I .
$$

Por tanto, se puede concluir que

$$
B^{d}=S \quad \text { y } \quad B^{s}=I .
$$

Entonces la tasa de interés es determinada tanto por el ahorro como por la inversión planeada.

\section{A.2 Preferencia por la liquidez}

Se introduce el mercado monetario en la ecuación que determina la tasa de interés, de acuerdo con la teoría de los fondos prestables. Es decir,

$$
B^{d}=S+\Delta M^{s} \quad B^{s}=I+\Delta M^{d},
$$

$o$ en equilibrio

$$
S(r)+\Delta M^{s}=I(r)+\Delta M^{d}(r) .
$$

En este modelo, se considera que la demanda de dinero se incrementa, por medio del aumento en la demanda por fondos prestables o en el flujo de oferta de bonos. En otras palabras, la demanda de financiamiento para invertir en bienes de capital, 'junto con la demanda por crédito de aquellos que desean o

18 La representación hace alusión a una economía cerrada. 
planean la reconstitución de su acervo de saldos monetarios, aumenta. Desde luego que un incremento en los saldos monetarios afecta proporcionalmente la demanda de bienes, pero esto se contempla en el término de ahorro $S$, ya que al aumentar el consumo cae el ahorro; de la misma forma que una emisión de bonos se puede utilizar para consumo, cancelándose en el agregado.

Ya que el ahorro y la inversión dependen de la tasa de interés, se puede suponer que la demanda de dinero está en función de la tasa de interés. Se supone además que la oferta monetaria es exógena. En este sentido, la ecuación anterior determina la tasa de interés de equilibrio, de tal manera que un aumento en la oferta monetaria reduce la tasa de interés y aumenta la demanda de bonos. El mercado de dinero y de bonos se equilibra, entonces, con un exceso de ahorro $S$ sobre la inversión $I$. Lo anterior significa que la tasa de interés es un fenómeno monetario, al menos parcialmente, ya que también influyen las fuerzas reales.

\section{A.3 La Ley de Walras}

Un planteamiento que incluye un modelo de equilibrio general se ejemplifica en la Ley de Walras, en la cual se establecen los siguientes mercados: a) bienes, b) ahorro e inversión, c) bonos y d) dinero. Las condiciones de equilibrio son:

$$
\begin{gathered}
C_{T}^{d}=C_{T}^{s}, \\
K_{T}^{d}=K_{T}^{s} \rightarrow K_{t+1}^{d}-K_{t}^{d}=K_{t+1}^{s}-K_{t}^{s}, \\
B_{T}^{d}=B_{T}^{s} \rightarrow B_{t+1}^{d}-B_{t}^{d}=B_{t+1}^{s}-B_{t}^{s}, \\
M_{T}^{d}=M_{T}^{s} \rightarrow M_{t+1}^{d}-M_{t}^{d}=M_{t+1}^{s}-M_{t}^{s},
\end{gathered}
$$

con las cuales se estable un equilibrio general de flujos.

Generalmente, la especificación de activos en los modelos de equilibrio parcial no aclara si el equilibrio alcanzado se mantiene desde el principio del período $(B O P)$ o si se trata de un equilibrio al final del período $(E O P)$. Más aún, en la determinación de este tipo de modelos, se utilizan indistintamente características como lo son las que se refieren a flujos y las que se refieren a stocks, asociadas tradicionalmente a las teorías de fondos prestables y de la preferencia por la liquidez, respectivamente.

Debido a que la descripción del mercado de bienes y de bonos es en flujos y la del mercado monetario es en stocks, habría que determinar si se puede hablar de una especificación de activos al principio o al final de periodo. Para ello, es necesario que exista primero compatibilidad entre los modelos de flujos y los de stocks, y posteriormente se puede plantear un equilibrio al principio o al final del período. 19

Ciertamente, el modelo de fondos prestables se construye con base en un equilibrio de flujos mientras que el de la preferencia por la liquidez se llevó a cabo en términos de stocks. Sin embargo, este último también se puede especificar en términos de flujos, de tal manera que el resultado final es equivalente con

19 Foley (1975). 
una especificación de final de período $(E O P)$, similar a la presentada para el equilibrio Walrasiano con las condiciones (8.A)-(11.A).

Consideremos que el tiempo puede dividirse en intervalos discretos $(t, t+1)$ y que los flujos se refieren a intervalos de tiempo $T$, donde $T$ es el intervalo de tiempo entre $t$ y $t+1$. Asimismo, denotemos a los saldos monetarios al principio de período como $M_{t}^{s}$ y en el último instante del período $T$, que es el inicio del período $t+1$, como $M_{t+1}^{s}$, o en otras palabras $\Delta M^{s}$. Por otra parte, los individuos toman decisiones en $t$ sobre los objetivos para cada uno de los stocks en el instante $t$ y $t+1$, o sobre los flujos en intervalo de tiempo $T$.

Los deseos de los consumidores e inversionistas respecto de sus saldos reales se llevan a cabo sobre el cambio de los mismos; similarmente a sus deseos sobre el ahorro o la adquicisión de capital físico, los cuales se realizan en función de su variación más el stock deseado en el instante $t+1$. De esta forma, los consumidores e inversionistas formulan sus objetivos en términos de stocks deseados de dinero, de capital físico y bonos en el instante $t$, que ellos desean mantener en el instante $t+1$. En este sentido un equilibrio de final de período hace compatible un equilibrio de flujos con el equilibrio de stocks.

Sin embargo, el equilibrio de principio de período implica que el ajuste se establece desde el instante $t$ y éste se mantiene a lo largo de todos los intervalos de tiempo. En este planteamiento surgen 2 preguntas: 1) si el equilibrio es permanente qué pasa. si surgen choques no anticipados que afecten la trayectoria de los saldos deseados con respecto a los existentes? En este caso, surgirían dos tipos de costos: uno por estar fuera del equilibrio y otro por el ajuste y 2) la implicación de que la velocidad de ajuste es instantánea es debatible, dado que los choques de información son asimétricos y se procesan en intervalos de tiempo diferente. ${ }^{20}$ En tales condiciones, el planteamiento correcto sería establecer un equilibrio general dentro de una esquema de final de período, el cual es compatible con el presentado en el modelo.

\section{Apéndice B}

\section{B.1 La teoría de las expectativas}

Según esta teoría, las tasas forward representan exclusivamente tasas futuras esperadas y la estructura intertemporal, en un momento del tiempo, refleja las expectativas actuales del mercado de una familia de tasas de interés futuras de corto plazo. Por ello, una pendiente positiva indica que el mercado espera que las tasas de corto plazo se eleven en un futuro próximo e inversamente si la pendiente es negativa. Si la pendiente es horizontal, indicará que las expectativas de las tasas futuras de corto plazo permanecerán relativamente constantes.

Los siguientes supuestos ilustran cómo las expectativas acerca de un aumento en las tasas futuras de corto plazo, lo cual se refleja en un incremento en la curva de rendimiento, afectan el comportamiento de los participantes dentro mercado. Lo anterior, asumiendo la existencia de una estructura intertemporal horizontal y que ante un choque de información económica, los participantes del mercado pueden anticipar el movimiento en las tasas de interés.

20 Véase Tapia (1990). 
a) Los participantes del mercado interesados en una inversión a largo plazo no desean la compra de bonos de largo plazo, ya que la expectativa de alza en las tasas de interés resultaría en una disminución de su precio y una pérdida de capital. Ellos preferirían invertir en corto plazo hasta que el aumento en las tasas se haya materializado, lo que les permitiría reinvertir sus fondos a una tasa mayor.

b) Los especuladores esperando una subida de tasas, anticipan un descenso en el precio de los bonos de largo plazo y desearán vender o irse en corto; de esta manera, los ingresos recibidos de la venta en corto de los bonos largos serán reinvertidos en instrumentos de deuda de corto plazo. Es decir, si se espera una subida de tasas, el precio de los bonos largos disminuirá, por lo que el especulador venderá esos bonos en corto y podría entonces comprarlos después a un precio menor para cubrir la venta en corto teniendo una ganancia.

c) Los demandantes de fondos desearán adquirir fondos de largo plazo jalando la parte final del mercado, ya que la competencia por estos fondos después será más cara.

Los casos anteriores tenderían a: 1) Bajar la demanda neta de bonos largos, aumentando parcialmente la oferta de bonos largos e 2) Incrementar la demanda de bonos cortos, lo cual elevaría los rendimientos de los bonos largos con relación a los cortos, desplazando la estructura de tasas consistente con la expectativa de incrementos futuros. A través de un razonamiento análogo, una expectativa de descenso de tasas, en respuesta a un choque de información económica, resultaría en una curva de rendimiento con pendiente negativa.

No obstante, se puede contra argumentar que si los mercados son eficientes y si las tasas forward predicen las tasas de interés futuras, entonces el precio de los bonos se conocería con exactitud sobre el horizonte de inversión, independientemente del plazo al vencimiento. Pero cuando hay incertidumbre sobre la trayectoria que tomarán las tasas de interés en el futuro y el precio de los bonos, estos instrumentos se convierten en inversiones riesgosas, ya que el riesgo varía proporcionalmente al plazo de vencimiento del activo. Algunos comentarios se pueden hacer al respecto para los administradores de fondos de inversión:

1) De acuerdo con la teoría pura de las expectativas, el retorno total esperado para cualquier período de inversión será igual sin importar el plazo de la estratégia seleccionada. ${ }^{21}$ Por ejemplo, si un inversionista tiene un horizonte a cinco años, no hará ninguna diferencia si compra un bono de cinco, diez ó veinte años, y lo mantiene cinco años. Debido a que el retorno total de los tres bonos será el mismo sobre los cinco años. Desde luego que el supuesto básico es que existe fungibilidad. ${ }^{22}$ No obstante, cabe aclarar que esta interpretación puede no ser válida generalmente, dados los riesgos asociados con la inversión en bonos largos.

2) Conocida como "Expectativas Locales" (EL). ${ }^{23}$ El retorno total será el mismo sobre un período de inversión de corto plazo empezando hoy. Por ejemplo, si un inversionista tiene un horizonte de inversión de seis meses, comprando

21 Lutz (1970).

22 Bonos que son intercambiables o equivalentes.

23 Cox, Ingersoll and Ross (1981). 
un bono de cinco, diez o veinte años producirá el mismo rendimiento total a seis meses. Esta es una de las interpretaciones probables que pueden ser sostenidas en equilibrio.

3) El de las "Expectativas Insesgadas" (EI). Si uno supone, como al principio, que las tasas forward son un pronóstico insesgado de las expectativas del mercado sobre las tasas futuras de interés. ${ }^{24}$

4) Otra interpretación sugiere que el retorno total que un inversionista puede llevar a cabo por el rolling over de bonos de corto plazo, sobre un horizonte de inversión, será lo mismo que mantener un $\mathrm{BCC}$ con vencimiento igual que el horizonte de inversión. Recordando que los BCC no tienen riesgo de reinversión, el futuro de las tasas de interés sobre el horizonte de inversión no afectará el retorno total.

5) Otra variante son las Expectativas del Retorno al Vencimiento (ERV). Por ejemplo, si suponemos que un inversionista tiene un horizonte de cinco años, comprando un $\mathrm{BCC}$ a cinco años y manteniéndolo hasta el vencimiento. $\mathrm{El}$ retorno total es la diferencia entre el valor al vencimiento y el precio del bono dividido entre el precio del bono. Es decir, comprando un instrumento a seis meses y haciendo rolling over en cinco años, el inversionista obtendrá el mismo retorno total.

Íntimamente ligado a esta interpretación está la teoría de la Expectativa del Rendimiento al Vencimiento (ERRV). Esta variante apunta que el retorno total será el mismo para cada período en el horizonte de inversión, sugiriendo que el retorno total al final del horizonte de inversión sea igual que el rolling over de instrumentos de corto plazo. Un inversionista con horizonte de inversión de cinco años, debería encontrar al final de seis meses, que el retorno total de mantener un BCC será igual que mantener un instrumento de seis meses. $\mathrm{Al}$ final de tres años, el retorno total de mantener el BCC de cinco años será igual que haber hecho un rolling over con un instrumento de seis meses por tres años. Cabe señalar que bajo condiciones especiales la interpretación de EI y de ERRV son iguales, lo cual sucede cuando el análisis ocurre en un período continuo.

\section{B.2 La teoría de la liquidez}

Bajo el enfoque de "Expectativas Puras" no se considera el riesgo asociado con la inversión en bonos; sin embargo, se puede demostrar que hay incertidumbre en el retorno de un período, por mantener un bono que tenga vencimiento mayor al período en cuestión, y la incertidumbre se incrementa sistemáticamente con la maduración del bono. ${ }^{25}$ Generalmente, a los inversionistas no les agrada el riesgo, de acuerdo con esto Hicks postuló que para inducir a los inversionistas a mantener vigencias o plazos mayores, es necesario ofrecer tasas de largo plazo mayores que el promedio esperado de las tasas futuras, por una prima de riesgo que aumente entre mayor sea el plazo. Es decir, que las tasas forward deberían reflejar ambos componentes, las expectativas de las tasas de interés y una prima de riesgo; por lo que las tasas forward implícitas, no serán un estimador insesgado de las expectativas del mercado acerca de las tasas de

24 Burton (1966).

25 Fabozzi and Modigliani (1992). 
interés en el futuro, ya que incluyen un premio por la liquidez. Una pendiente positiva puede reflejar expectativas sobre futuras tasas de interés, aunque la estructura de tasas sea creciente, igual o decreciente debido a un premio por liquidez que se incremente rápidamente con el plazo.

\section{B.3 El hábitat preferido}

Esta teoría formulada por Modigliani y Sutch adopta la visión de que la estructura de la tasa refleja la expectativa del sendero futuro de las tasas de interés, así como la prima de riesgo; pero rechaza el supuesto de que la prima de riesgo debe incrementarse uniformemente con el vencimiento. ${ }^{26}$ Lo anterior sería válido si todos los inversionistas intentan salirse del mercado en el menor tiempo posible y las contrapartes desean irse largos, pero la aversión al riesgo indica que solo se irá largo si este horizonte iguala al de la inversión planeada. Toda vez que si compran un instrumento de plazo menor tendrán que asumir el "Riesgo de Reinversión", o sea el riesgo de una caída en la tasa de interés al momento de reinvertir. Similarmente, si se compra un instrumento con maduración mayor al período de inversión se tendrá el riesgo dè una pérdida en el precio del activo, Risk price, de liquidar la posición antes del vencimiento.

La prudencia y la seguridad indican que el vencimiento entre activos y pasivos se iguala. Sin embargo, hay una preferencia en algunos sectores por determinados vencimientos. Las compañías de seguros emiten papel a cinco años garantizando los rendimientos y no están dispuestas a asumir un riesgo de reinversión si invierten a seis meses. Las cajas de ahorro están expuestas al riesgo de precios o de tasa de interés, ya que emiten a tasa fija o sus pasivos son a tasa fija, por ejemplo a un año e invierten a plazo de veinte años. Para ambos tipos de instituciones existe un riesgo al cambiar de vencimientos preferidos o "hábitat preferido", es decir, el que no exista una igualación en los plazos de vencimiento de la demanda y la oferta de fondos inducirá al cambio de vencimientos para balancear posiciones que necesitan compensarse por una prima de riesgo; la cual dependerá de la aversión al riesgo. En este sentido, la pendiente de la estructura intertemporal de tasas de interés estará determinada рог las expectativas sobre las tasas futuras de interés y la prima de riesgo; lo que induce a los participantes del mercado a cambiar de hábitat preferido. Esto último estará reflejado en la pendiente de la ETI, la cual puede ser positiva, negativa, horizontal o mixta.

\section{B.4 La segmentación de mercados}

Esta teoría también reconoce que los inversionistas tienen un hábitat preferido y que la razón principal de la pendiente de la EITI recae en las restricciones de la hoja de balance, es decir activos y pasivos, las cuales pueden ser de carácter regulatorio. Por ejemplo: a) De mercado (restricciones de crédito a plazos específicos) ${ }^{27}$ o b) Autoregulatorio.

Una diferencia sustantiva con la teoría del hábitat preferido es el supuesto de que ni los inversionistas ni los demandantes de crédito están dispuestos a

\footnotetext{
26 Modigliani and Sutch (1996).

27 Véase Culberston (1957).
} 
cambiar de un tipo de vencimientos a otro, para tomar ventaja de las oportunidades que surgen entre las expectativas y las tasas de interés forward, por lo que la pendiente de la EITI es determinada por la oferta y demanda de valores a plazo. Lo anterior presupone, la prevalencia de una absoluta aversión al riesgo. Esto es que los participantes tenderán a cambiar su hábitat preferido cuando existan discrepancias entre el mercado y las tasas esperadas, asegurándose que estas diferencias no sean muy grandes y no tiendan a ensancharse como para que incidan en el regreso al hábitat preferido.

\section{Apéndice $\mathbf{C}$}

La especificación en primeras diferencias establece la existencia de raíces unitarias en una serie de tiempo no estacionaria. Engle y Granger (1987) señalan que una combinación lineal de dos o mas series no estacionarias, puede llegar a ser estacionaria. Una combinación lineal estacionaria se interpreta como una relación de equilibrio de largo plazo entre las variables. El argumento tradicional es que las series endógenas que no están cointegradas, en el largo plazo diferirán de las variables cointegradas, permitiendo una variación muy amplia en el corto plazo.

Al término de cointegración se le conoce como término de corrección de errores, ya que la desviación del equilibrio de largo plazo es corregida gradualmente, a través de un mecanismo de ajuste parcial de corto plazo. Este es un modelo vectorial restringido, el cual contiene restricciones de cointegración en su especificación y se usa con series no estacionarias pero cointegrables, denominado vector de corrección de errores $(V E C)$.

La prueba de cointegración se llevó a cabo para un grupo de series noestacionarias. En caso de que sean cointegradas se deben identificar las relaciones de largo plazo, para ello se considera un VAR de orden " $p "$, tal que:

$$
y_{t}=A_{1} y_{t-1}+\cdots+A_{p} y_{t-p}+B x_{t}+\varepsilon_{t},
$$

donde $y_{t}$ es un vector " $k$ " no-estacionario de variables, $I(1) ; x_{t}$ es un vector de orden " $d$ " de variables deterministas y " $\varepsilon_{t}$ " es el vector de innovaciones. Por lo que el $V A R$ queda como:

$$
\Delta y=\Pi y_{t-1}+\sum_{I=1}^{p-1} \Gamma_{i} \Delta y_{t-1}+B x_{t}+\varepsilon_{t},
$$

donde

$$
\Pi=\sum_{I=1}^{p} A_{i}-I \quad \text { y } \quad \Gamma i=-\sum_{j=i+1}^{p} A_{j} .
$$

Si el coeficiente de la matriz $\Pi$ tiene un rango reducido $r<k$, entonces existen $k^{*} r$ matrices $\alpha$ y $\beta$ cada una con rango $r$, tal que $\Pi=\alpha \beta^{\prime}$ y $\beta^{\prime} y_{t}$ es estacionaria. $r$ es el número de relaciones cointegradas, o el rango de cointegrandos, y cada columna de $\beta$ es el vector de cointegrados. A los elementos de $\alpha$ se les denomina parámetros de ajuste.

Primero se estimará la matriz $\Pi$ sin restricciones y se probará si se rechazan o no las restricciones impuestas sobre el rango de $\Pi$. Para determinar el número 
de relaciones a cointegrar, si hay $k$ variables endógenas y cada una tiene una raíz unitaria, puede haber entonces desde cero hasta $k-1$ relaciones cointegrables linealmente independientes. En el vector de relaciones a cointegrar, se supone una tendencia determinista cuadrática de la siguiente forma:

$$
H^{*}(r): \Pi y_{t-1}+B x_{t}=\alpha\left(\beta^{\prime} y_{t-1}+\rho_{0}+\rho_{1} t\right)+\alpha_{1}\left(\gamma_{0}+\gamma_{1} t\right)
$$

en donde $\alpha_{1}$ es una matriz no única de $k^{*}(k-r)$, tal que $\alpha^{\prime} \alpha_{1}=0$ y el rango $\left(\left[\alpha \mid \alpha_{1}\right]\right)=k$. También se incluyó una variable dicotómica, la cual afectará desde luego la media y la tendencia de la serie. Los resultados, bajo el supuesto de una tendencia determinista cuadrática, son los siguientes:

$$
\begin{aligned}
& D L P-1.047 D L T C P E+0.706 D L W N+0.882 D L P E M-0.753 D L M 4 \\
& (0.571) \quad(0.603) \\
& -0.437 D L Y-1.361 D L P E P .
\end{aligned}
$$

Las estimaciones resultaron satisfactorias y la prueba del Likelihood Ratio: 1388.219 indica que todas las variables incluidas en la ecuación de la inflación son cointegradas y que no hay raíces unitarias. También hay que recordar que el ordenamiento de las variables del $V E C$ corresponden al ordenamiento de las variables en la ecuación de inflación.

\section{Apéndice D}

El deterioro de la especificación del modelo debido a cambios en el comportamiento histórico de las variables, puede ser verificado con la aplicación de pruebas de cambio estructural. Recordemos que este problema es posible remediarlo a medida que se aumenta el número de observaciones en la estimación del modelo, al mismo tiempo que se incrementa la eficiencia de los parámetros calculados. ${ }^{28}$ Para este efecto se utilizó la prueba de Chow de cambio estructural, basado en la comparación restringida y no restringida de la suma cuadrada de residuales:

$$
F=\frac{\left(u^{\prime} u-u_{1}^{\prime} u_{1}-u_{2}^{\prime} u_{2}\right) / k}{\left(u_{1}^{\prime} u_{1}+u_{2}^{\prime} u_{2}\right) /(T-2 k)} .
$$

La distribución es una $F_{t-2 k}^{k}$, de donde $u^{\prime} u$ es la suma cuadrada de residuales del modelo no restringido. $u_{1}^{\prime} u_{1}$ y $u_{2}^{\prime} u_{2}$ son las correspondientes a los sub-periodos uno y dos.

Con respecto al $C U S U M$, esta prueba se basa en la acumulación de la suma cuadrada de los residuales con una banda de 5\%. La prueba es:

$$
W_{t}=\sum_{r=k+1}^{t} \frac{w_{r}}{s}, \text { con } t=k+1, \cdots, T
$$

28 Salas (1990). 
donde $w_{r}$ es el residual recursivo, $s$ es el error estandar de la regresión ajustada en todos los puntos del tamaño muestral $T$. En este sentido, si el vector $\beta$ permanece constante de período en período tenemos que $E\left(W_{t}\right)=0$; sin embargo, si $\beta$ cambia entonces $W_{t}$ tenderá a diverger de la línea valor medio cero. La significancia de la divergencia está dada por las líneas de significancia al $5 \%$, es decir, la distancia entre la cual se incrementa con el paso del tiempo $t$. Los puntos de las bandas son:

$$
\left[k, \pm 0.948(T-k)^{1 / 2}\right] \quad \text { y } \quad\left[T, \pm 3^{*} 0.948(T-k)^{1 / 2}\right] .
$$

El movimiento de $W_{t}$ fuera de las líneas críticas (bandas superior e inferior) sugeriría una inestabilidad de los coeficientes.

\section{Bibliografía}

Aho, A. (1974). The Design, and Analysis of Computer Algorithms. Addison Wesley.

Burton, M. (1966). The Term Structure of Interest Rates: Expectations, and Behavior Patterns. Princeton University.

Cox, J., J. Ingersoll, and S. Ross (1981). A Re-examination of Traditional Hypotesis about the Term Structure of Interes Rate. Journal of Finance, pp. 769-799.

Culberston, J. M. (1957). The Term Structure of Interest Rates. Quaterly Journal of Economics, pp. 489-504.

Edwards, S. (1989). In Real Exchange Rates, Devaluation, and Adjustment. MIT Press.

Fabozzi, F., and F. Modigliani (1992). Capital Markets: Institutions, and Instruments. Prentice Hall.

Fabozzi, F. J. (1993). Bond Markets, Analysis, and Strategies. Prentice Hall.

Foley, D. K. (1975). On Two Specifications of Asset Equilibrium in Macroeconomic Models. Journal of Political Economy, 83, pp. 303-324.

Livingston, G. D. (1990). Bond Risk Analysis New York Institute of Finance. Informe Anual del Banco de México. Diversos números

Lutz, F. (1970). The Structure of Interest Rates. Quaterly Journal of Economics, pp. 36-63.

Modigliani, F,; and R. Sutch (1996). Innovations in Interest Rate Policy. American Economic Review, pp. 178-197.

Shiller, J. R., and J. H. McCulloch (1991). The Term Structure of Interest Rates. In Arrow, K. J., and M. D. Intriligator (Eds.). Handbook of Monetary Economics. Vol. I, North Holland, pp. 629-675

Salas, J. (1990). Econometría Aplicada a los Países en Desarrollo el Caso Mexicano. Fondo de Cultura Económica.

Tapia, J. (1985). Inflación, Causas y Efectos. Mimeo.

Tapia, J. (1990). Diferenciales de Tasas de Interés y la Paridad del Poder de Compra en Regímenes Cambiarios Flexibles: la Experiencia Mexicana 1978:01 1987:02. El Trimestre Económico, Julio-Septiembre.

Tapia, J. (1992). La Reacción de algunas Tasas de Interés ante Cambios de la Tasa Líder en una Economía Abierta. Un Análisis para México. Economía Mexicana, Nueva Época. 